\title{
Decentralized Control for the Interconnected Time-Delay Singular/Nonlinear Subsystems with Closed-Loop Decoupling Property
}

\author{
Nien-Tsu Hu, ${ }^{1}$ Ter-Feng Wu, ${ }^{2}$ Sendren Sheng-Dong Xu, ${ }^{3}$ and Pu-Sheng Tsai ${ }^{4}$ \\ ${ }^{1}$ Integrated Logistical Support Center, National Chung-Shan Institute of Science and Technology, Taoyuan 32599, Taiwan \\ ${ }^{2}$ Department of Electrical Engineering, National Ilan University, Ilan 26047, Taiwan \\ ${ }^{3}$ Graduate Institute of Automation and Control, National Taiwan University of Science and Technology, Taipei 10607, Taiwan \\ ${ }^{4}$ Department of Electronic Engineering, China University of Science and Technology, Taipei 11581, Taiwan
}

Correspondence should be addressed to Ter-Feng Wu; tfwu@niu.edu.tw

Received 26 September 2014; Accepted 4 December 2014

Academic Editor: Jun $\mathrm{Hu}$

Copyright (C) 2015 Nien-Tsu Hu et al. This is an open access article distributed under the Creative Commons Attribution License, which permits unrestricted use, distribution, and reproduction in any medium, provided the original work is properly cited.

\begin{abstract}
This paper presents the decentralized trackers using the observer-based suboptimal method for the interconnected time-delay singular/nonlinear subsystems with closed-loop decoupling property. The observer-based suboptimal method is used to guarantee the high-performance trajectory tracker for two different subsystems. Then, due to the high gain that resulted from the decentralized tracker, the closed-loop system will have the decoupling property. An illustrative example is given to demonstrate the effectiveness of the proposed control structure.
\end{abstract}

\section{Introduction}

The singular system model is a natural presentation of dynamic systems, such as power systems [1] and largescale systems $[2,3]$. In general, an interconnection of state variable subsystems is conveniently described as a singular system, even though an overall state space representation may not even exist. Over the past decades, much attention has been focused on the decentralized control [4-6] for timedelay singular systems. In [7], the problem of decentralized stabilization has been discussed for nonlinear singular largescale time-delay control systems with impulsive solutions. The $H_{\infty}$ control for singular systems with state delay has been presented in [8]. And the decentralized output feedback control problem [9] is considered for a class of large-scale systems with unknown time-varying delays.

In the recent years, a large number of control systems are characterized by interconnected large-scale subsystems, and many practical examples have been applied to decentralized control systems. The decentralized control of interconnected large-scale systems has commonly appeared in our modern technologies, such as transportation systems, power systems, and communication systems [10-12]. However, a survey of the literature indicates that the singular system issue has seldom been studied in such systems. Many research [1316] results concerning the singular/nonlinear system have successfully solved lots of complex problems. For the above reasons, we will discuss the decentralized control of the interconnected large-scale time-delay singular subsystem and nonlinear subsystem.

In this paper, we consider the time-delay effect. In practical applications, the time-delay effect [17-19] may result in an unexpected and unsatisfactory system performance, even including the serious instability, if it is ignored in the design of control systems. In order to overcome this problem, the controller design method $[20,21]$ is necessary to be further explored in this paper. Sequentially, the decentralized tracker with the high-gain property will make the closed-loop system own the decoupling property.

This paper is organized as follows. Section 2 describes the problem of interest. Section 3 presents the observer-based suboptimal digital tracker. Section 4 presents the simulation results of interconnected time-delay singular/nonlinear subsystems. Finally, Section 5 draws conclusions. 


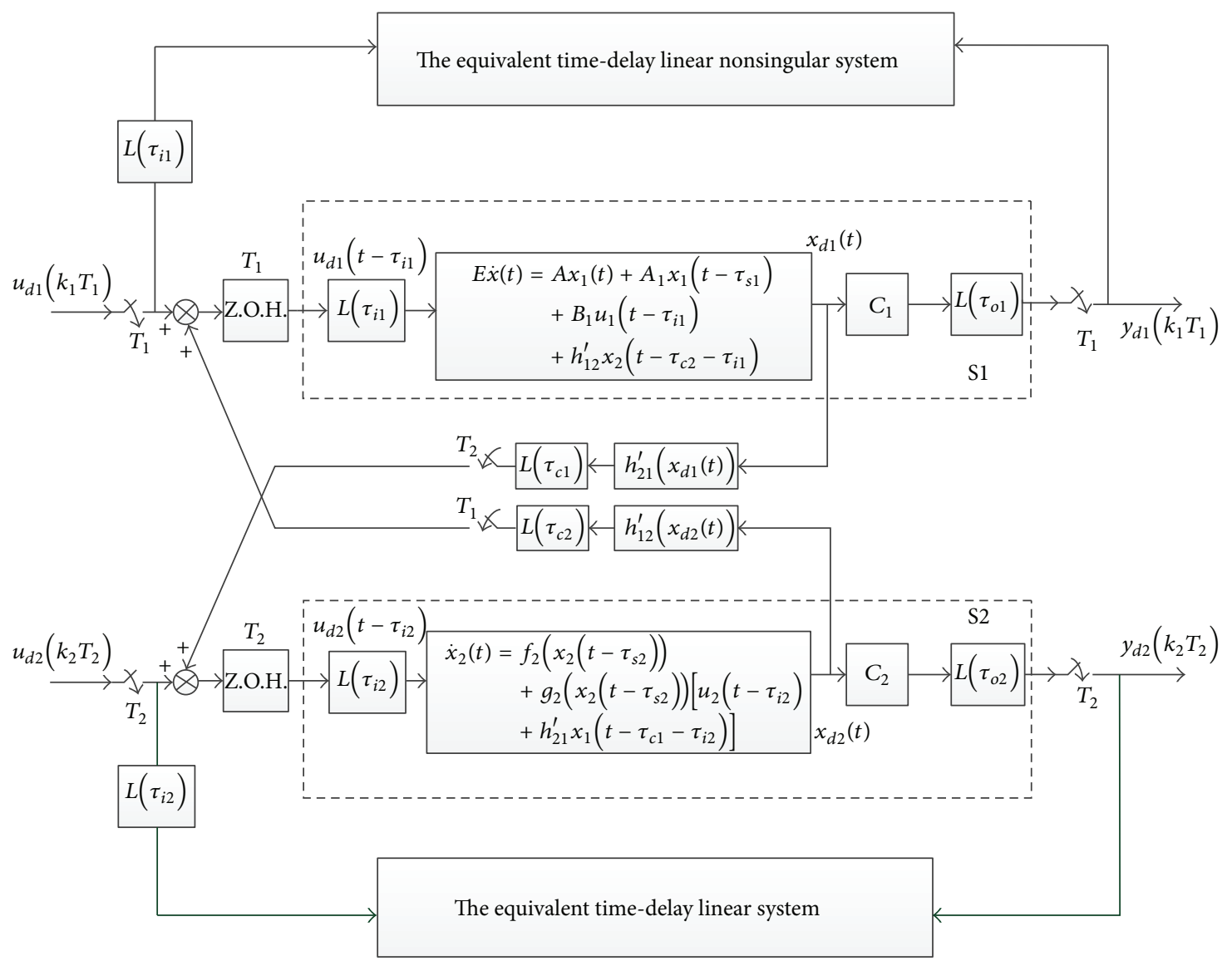

FIGURE 1: The schematic design methodology for the interconnected time-delay singular/nonlinear system.

\section{System and Problem Description}

Consider the time-delay system consisting of two interconnected MIMO subsystems shown as

$$
\begin{aligned}
S 1: \quad E \dot{x}(t)= & A x_{1}(t)+A_{1} x_{1}\left(t-\tau_{s 1}\right)+B_{1} u_{1}\left(t-\tau_{i 1}\right) \\
+ & h_{12}^{\prime} x_{2}\left(t-\tau_{c 2}-\tau_{i 1}\right) \\
& y_{1}(t)=C_{1} x_{1}\left(t-\tau_{o 1}\right) \\
\text { S2: } \quad \dot{x}_{2}(t)= & f_{2}\left(x_{2}\left(t-\tau_{s 2}\right)\right)+g_{2}\left(x_{2}\left(t-\tau_{s 2}\right)\right) \\
& \cdot\left[u_{2}\left(t-\tau_{i 2}\right)+h_{21}^{\prime} x_{1}\left(t-\tau_{c 1}-\tau_{i 2}\right)\right] \\
& y_{2}(t)=C_{2} x_{2}\left(t-\tau_{o 2}\right)
\end{aligned}
$$

where $x_{1}(t)$ and $x_{2}(t)$ are the state vectors, $u_{1}(t)$ and $u_{2}(t)$ are the control input vectors, and $y_{1}(t)$ and $y_{2}(t)$ are the output vectors. $f_{2}(\cdot)$ and $g_{2}(\cdot)$ are nonlinear functions of the states $x_{2}(t)$ of $S 2$. E, $A, A_{1}, B_{1}, C_{1}$, and $C_{2}$ are known as constant system matrices of appropriate dimensions and $E$ is a singular matrix. State time delays $\tau_{s 1}$ and $\tau_{s 2}$, interconnection time delays $\tau_{c 1}$ and $\tau_{c 2}$, input time delays $\tau_{i 1}$ and $\tau_{i 2}$, and output time delays $\tau_{o 1}$ and $\tau_{o 2}$ are assumed to be known. The time delays of interconnected state vectors $h_{12}^{\prime} x_{2}\left(t-\tau_{c 2}-\tau_{i 1}\right)$ and $h_{21}^{\prime} x_{1}\left(t-\tau_{c 1}-\tau_{i 2}\right)$ are induced from multiple sensors at different rates to accurately produce a reliable navigation solution.

The subsystem $S 1$ is the time-delay singular system and subsystem $S 2$ is the time-delay nonlinear subsystem. Before designing the controller, the decentralized modeling of the interconnected time-delay system is proposed in Figure 1. The notation $L(\cdot)$ through this paper is a time lag operator; for example, $L\left(\tau_{i j}\right) u(t)=u\left(t-\tau_{i j}\right)$.

It is very difficult to directly design the tracker and observer for $S 1$ and $S 2$ because their system models are not nonsingular and linear models. To solve this problem, the previously proposed method in [21] and the OKID (observer/Kalman filter identification) method in [22] are appropriately utilized to make $S 1$ and $S 2$ become the equivalent linear time-delay nonsingular subsystems. As a result, the process becomes quite simple. Besides, as long as the designed tracker for each subsystem has the high-gain property, the designed global system will have the closed-loop decoupling property.

We will use the proposed schematic design in Figure 1 to construct the methodology of the decentralized control for the interconnected time-delay singular/nonlinear subsystems with the closed-loop decoupling property.

\section{Main Results}

In this section, we construct the methodology of the decentralized control by using the design concept of the 
observer-based suboptimal digital tracker to control timedelay singular subsystem and time-delay nonlinear subsystem, respectively. Before designing the controller, we need to obtain the equivalent time-delay linear nonsingular subsystem and the equivalent time-delay linear subsystem. The problem of decentralized stabilization is discussed in the appendix.

3.1. The Equivalent Time-Delay Linear Nonsingular Subsystems for the Time-Delay Singular/Nonlinear Subsystems. From the schematic design methodology of Figure 1, and by using the previous method in [20], we can make the time-delay singular subsystems (1a) and (1b) become the equivalent time-delay regular system as follows:

$$
\begin{aligned}
& \dot{\tilde{x}}_{s}(t)= A_{s} \tilde{x}_{s}(t)+\widehat{A}_{d} \tilde{x}_{s}\left(t-\tau_{s 1}\right)+B_{d} v_{c}\left(t-\tau_{i 1}\right) \\
&+h_{12}^{\prime} x_{2}\left(t-\tau_{c 2}-\tau_{i 1}\right) \\
& y_{1}(t)=C_{11} \tilde{x}_{s}\left(t-\tau_{o 1}\right)-D_{1} v_{c}\left(t-\tau_{i 1}\right)
\end{aligned}
$$

where the parameters $A_{s}, \widehat{A}_{d}, B_{d}, C_{11}$, and $D_{1}$ and input $v_{c}(t)$ can be referred to in [20].

Remark A.0. Notably, definitions of the regular pencil [23] and the standard pencil [24] are satisfied on no state delay term in systems (1a) and (1b). If $A_{1}$ exists, then definitions of the regular pencil and the standard pencil do not guarantee that systems (1a) and (1b) can be decomposed into the equivalent time-delay regular system.

Similarly, the time-delay nonlinear subsystems (2a) and (2b) can transform the equivalent time-delay linear subsystem by OKID method [21, 22] as follows:

$$
\begin{gathered}
x_{d 2}\left(k_{2} T_{2}+T_{2}\right)=G_{d 2} x_{d 2}\left(k_{2} T_{2}\right)+H_{d 2} u_{d 2}\left(k_{2} T_{2}-\tau_{i 2}\right), \\
y_{d 2}\left(k_{2} T_{2}\right)=C_{d 2} x_{d 2}\left(k_{2} T_{2}-\tau_{o 2}\right),
\end{gathered}
$$

where $G_{d 2}, H_{d 2}$, and $C_{d 2}$ are the identified parameters by OKID method. The corresponding continuous-time system of ( $4 \mathrm{a})$ and $(4 \mathrm{~b})$ is described by

$$
\begin{gathered}
x_{c 2}(t)=A_{c 2} x_{c 2}(t)+B_{c 2} u_{2}\left(t-\tau_{i 2}\right), \\
y_{c 2}(t)=C_{c 2} x_{c 2}\left(t-\tau_{o 2}\right) .
\end{gathered}
$$

Notably, $A_{c 2}, B_{c 2}$, and $C_{c 2}$ are known as constant system matrices of appropriate dimensions.

The equivalent subsystems (3a), (3b), and (5a) and (5b) will be applied to the observer-based suboptimal digital tracker [21] for the singular/nonlinear subsystem in the next subsection and finally we proposed the schematic design methodology of decentralized control for the interconnected time-delay singular/nonlinear subsystems with closed-loop decoupling property.

3.2. The Observer-Based Suboptimal Digital Tracker Design [21]. Consider the continuous time-delay singular subsystems (3a) and (3b) or the time-delay subsystems (5a) and (5b). Here, we take the time-delay singular subsystems (3a) and $(3 b)$ to design the observer-based suboptimal digital tracker and the design results are similar to the time-delay subsystems (5a) and (5b).

Consider the continuous time-delay singular subsystems (3a) and (3b) without the time delay of interconnected state vector $h_{12}^{\prime} x_{2}\left(t-\tau_{c 2}-\tau_{i 1}\right)$. By [21], $T_{1}$ is the sampling period. Let the state delay time be given by $\tau_{s 1}=\rho_{1} T_{1}+\Gamma_{1}$, where $0 \leq \Gamma_{1}<T_{1}$ and $\rho_{1} \geq 0$ is an integer, and let the input delay time be given by $\tau_{i 1}=\eta_{1} T_{1}+\sigma_{1}$, where $0 \leq \sigma_{1}<T_{1}$ and $\eta_{1} \geq 0$ is an integer. The time-delay singular subsystems (3a) and ( $3 b)$, by both the Newton extrapolation method and the Chebyshev quadrature method $[25,26]$, become

$$
\begin{aligned}
\tilde{x}_{d s}( & \left.(k+1) T_{1}\right) \\
= & G \widetilde{x}_{d s}\left(k T_{1}\right)+\widehat{G}_{1}^{(1)} \widetilde{x}_{d s}\left(k T_{1}-\rho_{1} T_{1}+T_{1}\right) \\
& +\widehat{G}_{1}^{(2)} \tilde{x}_{d s}\left(k T_{1}-\rho_{1} T_{1}\right)+\widehat{G}_{1}^{(3)} \tilde{x}_{d s}\left(k T_{1}-\rho_{1} T_{1}-T_{1}\right) \\
& +H_{1}^{(0)} v_{d}\left(k T_{1}-\eta_{1} T_{1}\right)+H_{1}^{(1)} v_{d}\left(k T_{1}-\eta_{1} T_{1}-T_{1}\right),
\end{aligned}
$$

where

$$
\begin{gathered}
G=e^{A_{s} T}, \quad \widehat{G}_{1}^{(1)}=\frac{T_{1}}{2}\left[Q_{1}^{(2)}+Q_{1}^{(3)}\right] \widehat{A}_{d}, \\
\widehat{G}_{1}^{(2)}=T_{1}\left[Q_{1}^{(1)}-Q_{1}^{(3)}\right] \widehat{A}_{d}, \quad \widehat{G}_{1}^{(3)}=\frac{T_{1}}{2}\left[Q_{1}^{(3)}-Q_{1}^{(2)}\right] \widehat{A}_{d}, \\
H_{1}^{(0)}=\left[G^{1-\gamma_{1}}-I_{n}\right] A_{s}^{-1} B_{d}, \quad H_{1}^{(1)}=\left[G-G^{1-\gamma_{1}}\right] A_{s}^{-1} B_{d},
\end{gathered}
$$

in which

$$
\begin{gathered}
\gamma_{1}=\frac{\sigma_{1}}{T_{1}}, \quad \beta_{1}=\frac{\Gamma_{1}}{T_{1}}, \quad Q_{1}^{(1)}=\left[G-I_{n}\right]\left(A_{s} T_{1}\right)^{-1}, \\
Q_{1}^{(2)}=\left[Q_{1}^{(1)}-\left(1-\beta_{1}\right) I_{n}-\beta_{1} G\right]\left(A_{s} T_{1}\right)^{-1}, \\
Q_{1}^{(3)}=\left[2 Q_{1}^{(2)}-\left(1-\beta_{1}\right)^{2} I_{n}-\beta_{1}^{2} G\right]\left(A_{s} T_{1}\right)^{-1} .
\end{gathered}
$$

Some terms in (6) may be combined because of the same delay, so (6) can be reduced to

$$
\begin{aligned}
\tilde{x}_{d s}\left((k+1) T_{1}\right) & \\
= & \bar{G} \tilde{x}_{d s}\left(k T_{1}\right)+\sum_{i=1}^{M_{1}} \bar{G}_{i} \widetilde{x}_{d s}\left(k T_{1}-i T_{1}\right) \\
& +\bar{H} v_{d}\left(k T_{1}\right)+\sum_{j=1}^{M_{2}} \bar{H}_{j} v_{d}\left(k T_{1}-j T_{1}\right) .
\end{aligned}
$$


The time-delay state $x_{1}\left(t-\tau_{o 1}\right)$ for $k T_{1} \leq t-\tau_{o 1}<(k+1) T_{1}$ must be evaluated as follows:

$$
\begin{aligned}
\tilde{x}_{s}(t- & \left.\tau_{o 1}\right) \\
= & e^{A_{s}\left(t-\tau_{o 1}-k T_{1}\right)} \tilde{x}_{d s}\left(k T_{1}\right) \\
& +\int_{k T_{1}}^{t-\tau_{o 1}} e^{A_{s}\left(t-\tau_{o 1}-\lambda\right)} \widehat{A}_{d} \tilde{x}_{d s}\left(\lambda-\tau_{s 1}\right) d \lambda \\
& +\int_{k T_{1}}^{t-\tau_{o 1}} e^{A_{s}\left(t-\tau_{o 1}-\lambda\right)} B_{d} v_{d}\left(\lambda-\tau_{i 1}\right) d \lambda \\
= & \delta_{1}\left(t-\tau_{o 1}-k T_{1}\right) \tilde{x}_{d s}\left(k T_{1}\right) \\
& +\left[\widehat{\delta}_{1}^{(1)}\left(t-\tau_{o 1}-k T_{1}\right) \tilde{x}_{d s}\left(k T_{1}-\rho_{1} T_{1}+T_{1}\right)\right. \\
& \quad+\widehat{\delta}_{1}^{(2)}\left(t-\tau_{o 1}-k T_{1}\right) \tilde{x}_{d s}\left(k T_{1}-\rho_{1} T_{1}\right) \\
& \left.\quad+\widehat{\delta}_{1}^{(3)}\left(t-\tau_{o 1}-k T_{1}\right) \tilde{x}_{d s}\left(k T_{1}-\rho_{1} T_{1}-T_{1}\right)\right] \\
& +\left[\varphi_{1}^{(0)}\left(t-\tau_{o 1}-k T_{1}\right) v_{d}\left(k T_{1}-\eta_{1} T_{1}\right)\right.
\end{aligned}
$$

where

$$
\begin{gathered}
\delta_{1}\left(t-\tau_{o 1}-k T_{1}\right)=e^{A_{s}\left(t-\tau_{o 1}-k T_{1}\right)}, \\
\widehat{\delta}_{1}^{(1)}\left(t-\tau_{o 1}-k T_{1}\right)=\frac{T_{1}}{2}\left[q_{1}^{(2)}+q_{1}^{(3)}\right] \widehat{A}_{d}, \\
\widehat{\delta}_{1}^{(2)}\left(t-\tau_{o 1}-k T_{1}\right)=T_{1}\left[q_{1}^{(1)}-q_{1}^{(3)}\right] \widehat{A}_{d}, \\
\widehat{\delta}_{1}^{(3)}\left(t-\tau_{o 1}-k T_{1}\right)=\frac{T_{1}}{2}\left[q_{1}^{(3)}-q_{1}^{(2)}\right] \widehat{A}_{d}, \\
\varphi_{1}^{(0)}\left(t-\tau_{o 1}-k T_{1}\right) \\
= \begin{cases}O_{n \times m}, & t-\tau_{o 1}<\sigma_{1} \\
{\left[e^{-A_{s} \sigma_{1}} \delta_{1}\left(t-\tau_{o 1}-k T_{1}\right)-I_{n}\right] A_{s}^{-1} B_{d},} & t-\tau_{o 1} \geq \sigma_{1},\end{cases} \\
\varphi_{1}^{(1)}\left(t-\tau_{o 1}-k T_{1}\right) \\
= \begin{cases}{\left[\delta_{1}\left(t-\tau_{o 1}-k T_{1}\right)-I_{n}\right] A_{s}^{-1} B_{d},} & t-\tau_{o 1}<\sigma_{1} \\
\delta_{1}\left(t-\tau_{o 1}-k T_{1}\right)\left[I_{n}-e^{-A_{s} \sigma_{1}}\right] A_{s}^{-1} B_{d}, & t-\tau_{o 1} \geq \sigma_{1},\end{cases}
\end{gathered}
$$

in which

$$
\begin{aligned}
q_{1}^{(1)}=[ & \left.\delta_{1}\left(t-\tau_{o 1}-k T_{1}\right)-I_{n}\right]\left(A_{s} T_{1}\right)^{-1}, \\
q_{1}^{(2)}=[ & q_{1}^{(1)}-\left(\frac{t-\tau_{o 1}-k T_{1}}{T_{1}}-\beta_{1}\right) I_{n} \\
& \left.-\beta_{1} \delta_{1}\left(t-\tau_{o 1}-k T_{1}\right)\right]\left(A_{s} T_{1}\right)^{-1},
\end{aligned}
$$

$$
\begin{aligned}
q_{1}^{(3)}=\left[2 q_{1}^{(2)}-\left(\frac{t-\tau_{o 1}-k T_{1}}{T_{1}}-\beta_{1}\right)^{2} I_{n}\right. \\
\left.+\beta_{1}^{2} \delta_{1}\left(t-\tau_{o 1}-k T_{1}\right)\right]\left(A_{s} T_{1}\right)^{-1} .
\end{aligned}
$$

Some terms in (10) may be combined as in (9), and (10) can be rewritten as

$$
\begin{aligned}
\tilde{x}_{s}\left(t-\tau_{o 1}\right)= & \bar{\delta}_{0}\left(t-\tau_{o 1}-k T_{1}\right) \tilde{x}_{d s}\left(k T_{1}\right) \\
& +\sum_{i=1}^{M_{1}} \bar{\delta}_{i}\left(t-\tau_{o 1}-k T_{1}\right) \tilde{x}_{d s}\left(k T_{1}-i T_{1}\right) \\
& +\bar{\varphi}_{0}\left(t-\tau_{o 1}-k T_{1}\right) v_{d}\left(k T_{1}\right) \\
& +\sum_{j=1}^{M_{2}} \bar{\varphi}_{j}\left(t-\tau_{o 1}-k T_{1}\right) v_{d}\left(k T_{1}-j T_{1}\right) .
\end{aligned}
$$

Then, the output (3b) can be rewritten as

$$
\begin{aligned}
y_{1}(t)= & C_{11} \tilde{x}_{s}\left(t-\tau_{o 1}\right)-D_{1} v_{c}\left(t-\tau_{i 1}\right) \\
= & C_{11} \bar{\delta}_{0}\left(t-\tau_{o 1}-k T_{1}\right) \tilde{x}_{d s}\left(k T_{1}\right) \\
& +\sum_{i=1}^{M_{1}} C_{11} \bar{\delta}_{1}\left(t-\tau_{o 1}-k T_{1}\right) \tilde{x}_{d s}\left(k T_{1}-i T_{1}\right) \\
& +C_{11} \bar{\varphi}_{0}\left(t-\tau_{o 1}-k T_{1}\right) v_{d}\left(k T_{1}\right) \\
& +\sum_{j=1}^{M_{2}} C_{11} \bar{\varphi}_{j}\left(t-\tau_{o 1}-k T_{1}\right) v_{d}\left(k T_{1}-j T_{1}\right) \\
& -\left[D_{1}^{(0)} v_{d}\left(k T_{1}-\eta_{1} T_{1}\right)+D_{1}^{(1)} v_{d}\left(k T_{1}-\eta_{1} T_{1}-T_{1}\right)\right],
\end{aligned}
$$

where $D_{1}^{(0)}=D_{1}^{*}\left(B_{d}^{T} B_{d}\right)^{-1} H_{1}^{(0)}, D_{1}^{(1)}=D_{1}^{*}\left(B_{d}^{T} B_{d}\right)^{-1} H_{1}^{(1)}$, and $D_{1}^{*}=\left[\begin{array}{ll}D_{1} & O\end{array}\right]^{T}$.

Similarly, some terms in (14) can be combined so (14) can be rewritten as

$$
\begin{aligned}
y_{1}(t)= & C_{11} \bar{\delta}_{0}\left(t-\tau_{o 1}-k T_{1}\right) \tilde{x}_{d s}\left(k T_{1}\right) \\
& +\sum_{i=1}^{M_{1}} C_{11} \bar{\delta}_{1}\left(t-\tau_{o 1}-k T_{1}\right) \tilde{x}_{d s}\left(k T_{1}-i T_{1}\right) \\
& +C_{11} \bar{\varphi}_{0}^{*}\left(t-\tau_{o 1}-k T_{1}\right) v_{d}\left(k T_{1}\right) \\
& +\sum_{j=1}^{M_{2}} C_{11} \bar{\varphi}_{j}^{*}\left(t-\tau_{o 1}-k T_{1}\right) v_{d}\left(k T_{1}-j T_{1}\right),
\end{aligned}
$$

where $\bar{\varphi}_{0}^{*}, \bar{\varphi}_{j}^{*}, \bar{\varphi}_{0}$, and $\bar{\varphi}_{j}$ are the summation of multiple inputdelay terms.

In the following work, we use (13) and (15) to derive the equivalent extended delay-free system as follows:

$$
\begin{gathered}
X_{d}\left((k+1) T_{1}\right)=\widehat{G}_{e} X_{d}\left(k T_{1}\right)+\widehat{H}_{e} v_{d}\left(k T_{1}\right), \\
y_{d}\left(k T_{1}\right)=\widehat{C}_{e} X_{d}\left(k T_{1}\right),
\end{gathered}
$$


where

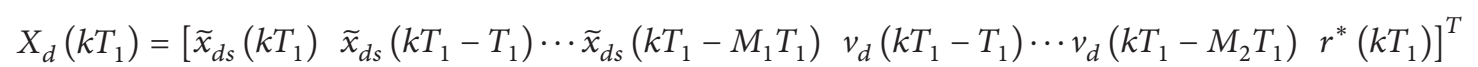

means the extended virtual state vector.

By the previous method [21], we derive the observerbased suboptimal tracker for the time-delay singular system with unavailable states using the equivalent extended delayfree system. The extended observer-based suboptimal digital tracker can be represented as

$$
\begin{aligned}
& \widehat{X}_{d}\left((k+1) T_{1}\right) \\
& =\widehat{G}_{o} \widehat{X}_{d}\left(k T_{1}\right)+\widehat{H}_{o} v_{d}\left(k T_{1}\right)+L_{d}\left[y_{d}\left(k T_{1}\right)-\widehat{C}_{e} \widehat{X}_{d}\left(k T_{1}\right)\right], \\
& v_{d}\left(k T_{1}\right)=-\widehat{K}\left(k T_{1}\right) \widehat{X}_{d}\left(k T_{1}\right),
\end{aligned}
$$

where $\widehat{X}_{d}\left(k T_{1}\right)$ is the estimate of the extended state $X_{d}\left(k T_{1}\right)$ in (17) and

$$
\begin{gathered}
\widehat{G}_{o}=\widehat{G}_{e}-L_{d} \widehat{C}_{e} \widehat{G}_{e}, \\
\widehat{H}_{o}=\widehat{H}_{e}-L_{d} \widehat{C}_{e} \widehat{H}_{e}, \\
\widehat{K}\left(k T_{1}\right)=\left[\begin{array}{lll}
K_{d}\left(k T_{1}\right) & F_{d}\left(k T_{1}\right) E_{d}\left(k T_{1}\right)
\end{array}\right],
\end{gathered}
$$

in which

$$
\begin{gathered}
K_{d}\left(k T_{1}\right)=\left[\begin{array}{lllll}
K_{d}^{(0)}\left(k T_{1}\right) & K_{d}^{(1)}\left(k T_{1}\right) & \cdots & K_{d}^{\left(M_{1}\right)}\left(k T_{1}\right)
\end{array}\right], \\
F_{d}\left(k T_{1}\right)=\left[\begin{array}{lllll}
F_{d}^{(1)}\left(k T_{1}\right) & \cdots & F_{d}^{\left(M_{2}\right)}\left(k T_{1}\right)
\end{array}\right] .
\end{gathered}
$$

The details of the parameters can be referred to in [21]. Here, the observer-based suboptimal tracker has been completely obtained. Figure 2 presents the realization of decentralized control for the interconnected time-delay singular/nonlinear subsystems.

From Figures 1 and 2, the design procedure can be summarized as the following steps.

Step 1. Perform the previously proposed method [21] and the OKID method [22] to determine the equivalent time-delay linear subsystems in Figure 1.

Step 2. Design the observer-based suboptimal digital trackers from the equivalent time-delay linear subsystems obtained in Step 1.

Step 3. Perform the observer-based suboptimal digital trackers obtained in Step 2. The decentralized control for the interconnected time-delay singular/nonlinear subsystems is shown in Figure 2.

\section{An Illustrative Example}

Consider the time-delay system consisting of two interconnected MIMO subsystems shown as

S1: $\quad E \dot{x}(t)=A x_{1}(t)+A_{1} x_{1}\left(t-\tau_{s 1}\right)+B_{1} u_{1}\left(t-\tau_{i 1}\right)$

$$
+h_{12}^{\prime} x_{2}\left(t-\tau_{c 2}-\tau_{i 1}\right)
$$

$$
y_{1}(t)=C_{1} x_{1}\left(t-\tau_{o 1}\right),
$$

$S 2: \quad \dot{x}_{2}(t)=f_{2}\left(x_{2}\left(t-\tau_{s 2}\right)\right)+g_{2}\left(x_{2}\left(t-\tau_{s 2}\right)\right)$

$$
\begin{gathered}
\cdot\left[u_{2}\left(t-\tau_{i 2}\right)+h_{21}^{\prime} x_{1}\left(t-\tau_{c 1}-\tau_{i 2}\right)\right], \\
y_{2}(t)=C_{2} x_{2}\left(t-\tau_{o 2}\right),
\end{gathered}
$$

where

$$
\begin{array}{ll}
u_{1}(t)=\left[\begin{array}{l}
u_{1,1}(t) \\
u_{1,2}(t)
\end{array}\right], & u_{2}(t)=\left[\begin{array}{l}
u_{2,1}(t) \\
u_{2,2}(t)
\end{array}\right], \\
x_{1}(t)=\left[\begin{array}{l}
x_{1,1}(t) \\
x_{1,2}(t) \\
x_{1,3}(t) \\
x_{1,4}(t) \\
x_{1,5}(t) \\
x_{1,6}(t)
\end{array}\right], & x_{2}(t)=\left[\begin{array}{l}
x_{2,1}(t) \\
x_{2,2}(t) \\
x_{2,3}(t) \\
x_{2,4}(t)
\end{array}\right] .
\end{array}
$$

The first subsystem $S 1$ of the large-scale system is given as follows:

$$
E=\left[\begin{array}{llllll}
1 & 2 & 1 & 1 & -3 & -2 \\
0 & 2 & 2 & 1 & -3 & -3 \\
1 & 2 & 1 & 1 & -3 & -2 \\
1 & 2 & 1 & 3 & -5 & -4 \\
0 & 2 & 1 & 1 & -2 & -2 \\
1 & 0 & 0 & 0 & -1 & 0
\end{array}\right], \quad A=I_{6}
$$




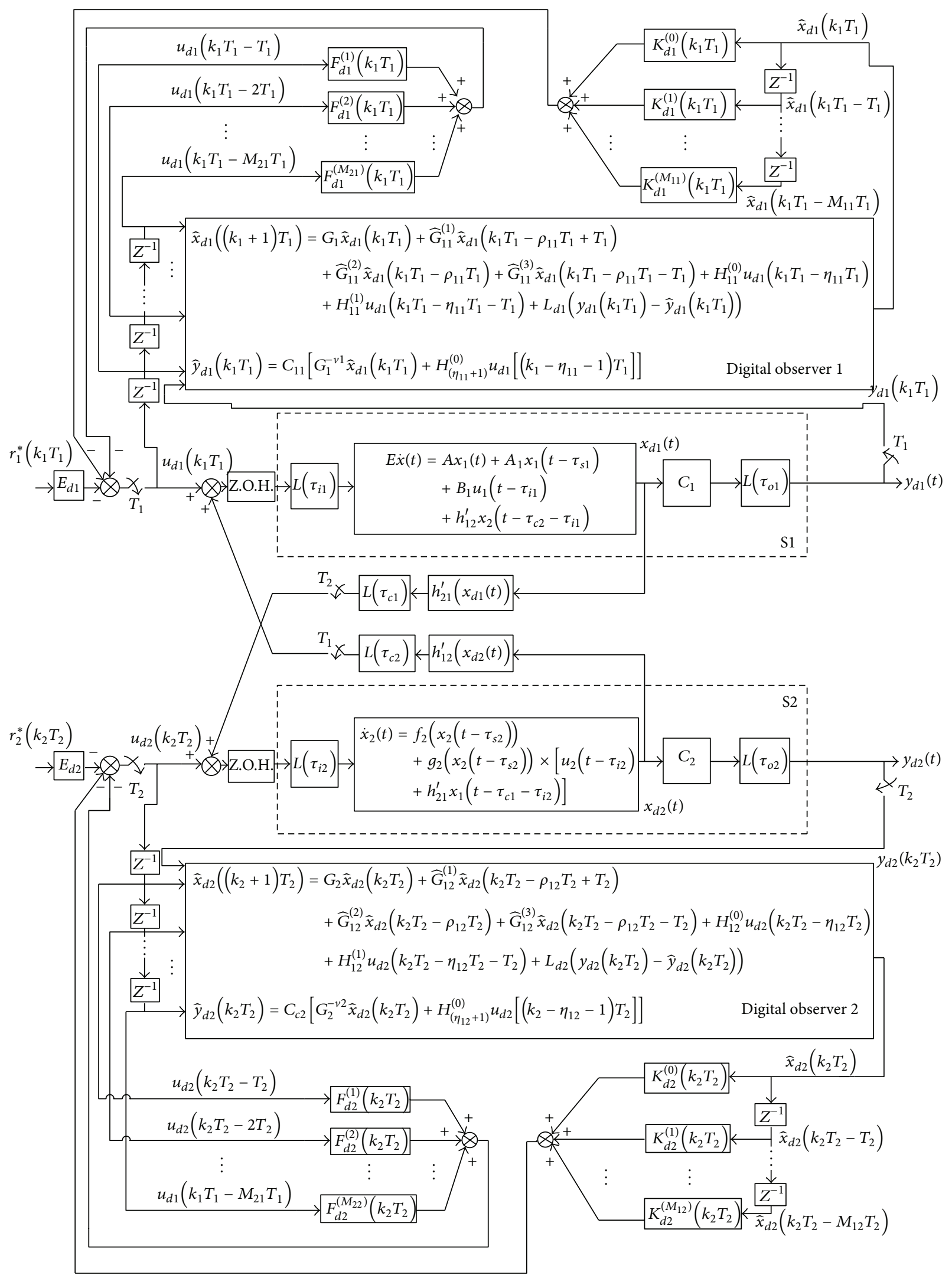

FIGURE 2: The decentralized control for the interconnected time-delay singular/nonlinear subsystems. 


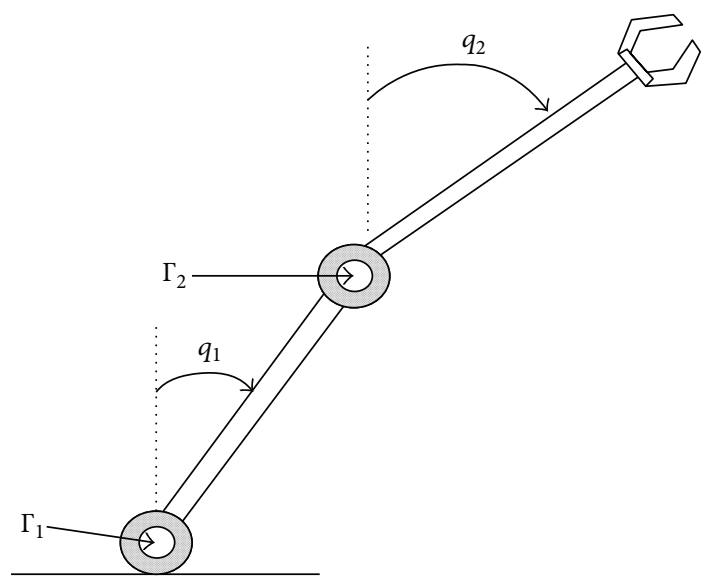

FIgURE 3: Two-link robot.

$A_{1}$

$$
\begin{aligned}
& =\left[\begin{array}{cccccc}
1.6207 & 0 & 0 & 0 & -1.6207 & 0 \\
0 & 0.8103 & -0.4052 & 0 & -1.6207 & 0 \\
0 & 0 & 0.8103 & 0 & 0 & 0 \\
0 & 0 & 0 & 1.6207 & -3.2413 & 0 \\
0 & 0 & 0 & 0 & 1.6207 & 0 \\
0 & 0 & 0 & 0 & -3.2413 & 1.6207
\end{array}\right] \text {, } \\
& B_{1}=\left[\begin{array}{cccccc}
1 & 0 & 0 & 0 & 0 & -1 \\
0 & 0 & -1 & 1 & 0 & 0
\end{array}\right]^{T}, \\
& C_{1}=\left[\begin{array}{llllll}
1 & 0 & 1 & 0 & 0 & 0 \\
0 & 1 & 0 & 1 & 0 & 0
\end{array}\right], \\
& \tau_{s 1}=0, \quad \tau_{i 1}=\tau_{o 1}=0.5 \times T_{1} .
\end{aligned}
$$

Let the sampling period $T_{1}=0.01 \mathrm{sec}$ and the initial condition is $x_{c}(0)=\left[\begin{array}{llllll}0 & 0 & 0 & 0 & 0 & 0\end{array}\right]^{T}$.

The second subsystem $S 2$ of the large-scale system is given by two-link robot $[27,28]$, which is described as shown in Figure 3.

The dynamic equation of the two-link robot system can be expressed as follows:

$$
M(q) \ddot{q}+C(q, \dot{q}) \dot{q}+G(q)=\Gamma,
$$

where

$$
\begin{gathered}
M(q)=\left[\begin{array}{cc}
\left(m_{1}+m_{2}\right) l_{1}^{2} & m_{2} l_{1} l_{2}\left(s_{1} s_{2}+c_{1} c_{2}\right) \\
m_{2} l_{1} l_{2}\left(s_{1} s_{2}+c_{1} c_{2}\right) & m_{2} l_{2}^{2}
\end{array}\right], \\
C(q, \dot{q})=m_{2} l_{1} l_{2}\left(c_{1} s_{2}-s_{1} c_{2}\right)\left[\begin{array}{cc}
0 & -\dot{q}_{2} \\
-\dot{q}_{1} & 0
\end{array}\right], \\
G(q)=\left[\begin{array}{c}
-\left(m_{1}+m_{2}\right) l_{1} g_{r} s_{1} \\
-m_{2} l_{2} g_{r} s_{2}
\end{array}\right],
\end{gathered}
$$

and $q=\left[\begin{array}{ll}q_{1} & q_{2}\end{array}\right]^{T}, q_{1}, q_{2}$ are the angular positions, $M(q)$ is the moment of inertia, $C(q, \dot{q})$ includes Ceoriolis and centripetal forces, $G(q)$ is the gravitational force, and $\Gamma$ is the applied torque vector. Here, we use the short hand notations $s_{i}=$ $\sin \left(q_{i}\right)$ and $c_{i}=\cos \left(q_{i}\right)$. The nominal parameters of the system are given as follows: the link masses $m_{1}=5 \mathrm{~kg}, m_{2}=2.5 \mathrm{~kg}$, the length $l_{1}=l_{2}=0.5 \mathrm{~m}$, and the gravitational acceleration $g_{r}=9.81 \mathrm{~ms}^{-2}$. Rewrite (25) in the following form:

$$
\ddot{q}=M^{-1}(q)(\Gamma-C(q, \dot{q}) \dot{q}-G(q)) .
$$

Let $x_{1}$ and $f_{1}\left(x_{1}\right)$ represent the state of the system and the nonlinear function of the state $x_{1}$, respectively. And the notation is shown as follows:

$$
\begin{gathered}
x_{2}(t) \equiv\left[\begin{array}{llll}
x_{2,1} & x_{2,2} & x_{2,3} & x_{2,4}
\end{array}\right]^{T}=\left[\begin{array}{llll}
q_{1} & \dot{q}_{1} & q_{2} & \dot{q}_{2}
\end{array}\right]^{T}, \\
f_{2}\left(x_{2}(t)\right) \equiv\left[\begin{array}{llll}
f_{2,1} & f_{2,2} & f_{2,3} & f_{2,4}
\end{array}\right]^{T},
\end{gathered}
$$

where $f_{2,1}=x_{2,2}, f_{2,3}=x_{2,4}$, and $\left[\begin{array}{ll}f_{2,2} & f_{2,4}\end{array}\right]^{T}=$ $M^{-1}\left(-C\left[\begin{array}{ll}x_{2,2} & x_{2,4}\end{array}\right]^{T}-G\right)$. Also, let $u_{2} \equiv \Gamma$, in which $\Gamma=$ $\left[\begin{array}{ll}\Gamma_{1} & \Gamma_{2}\end{array}\right]^{T}$.

Calculate the inverse of the matrix $M$, and then we can have $M^{-1}=\left[\begin{array}{ll}p_{11} & p_{12} \\ p_{21} & p_{22}\end{array}\right]$ such that

$$
g_{2}\left(x_{2}(t)\right)=\left[\begin{array}{cc}
0 & 0 \\
p_{11} & p_{12} \\
0 & 0 \\
p_{21} & p_{22}
\end{array}\right] .
$$

Therefore, the dynamic equation of the two-link robot system can be reformulated as follows:

$$
\begin{gathered}
\dot{x}_{2}(t)=f_{2}\left(x_{2}\left(t-\tau_{s 2}\right)\right)+g_{2}\left(x_{2}\left(t-\tau_{s 2}\right)\right) u_{2}\left(t-\tau_{i 2}\right), \\
y_{2}(t)=C_{2} x_{2}\left(t-\tau_{o 2}\right),
\end{gathered}
$$

where $C_{2}=\left[\begin{array}{llll}1 & 0 & 0 & 0 \\ 0 & 0 & 1 & 0\end{array}\right]$, the sampling period $T_{2}=0.02 \mathrm{sec}$, and the initial condition $x_{2}(0)=\left[\begin{array}{llll}0 & 0 & 0 & 0\end{array}\right]^{T}$.

Combining the above systems with the nonlinear interconnected terms, the large-scale system can then be shown in Figures 1 and 2, where the nonlinear interconnected terms $h_{12}^{\prime}\left(x_{d 2}(t)\right)$ and $h_{21}^{\prime}\left(x_{d 1}(t)\right)$ are given as $\left[\begin{array}{c}x_{d 2,3}^{2} \cos \left(x_{d 2,1}\right) \\ \sin ^{2}\left(x_{d 2,2}\right)\end{array}\right]$ and $\left[\begin{array}{c}x_{d 1,1}^{2} \\ x_{d 1,3} \sin \left(x_{d 1,2}\right)\end{array}\right]$, respectively. The time delays of the nonlinear interconnected terms are $\tau_{c 1}=3 \times T_{2}$ and $\tau_{c 2}=1 \times T_{1}$, where $T_{1}=0.01 \mathrm{sec}$ and $T_{2}=0.02 \mathrm{sec}$.

Based on Section 3.1 [20], the time-delay singular subsystem $S 1$ can be transformed into the equivalent time-delay regular system as follows:

$$
\begin{aligned}
S 1: \quad \dot{\tilde{x}}_{s}(t)= & A_{s} \widetilde{x}_{s}(t)+\widehat{A}_{d} \tilde{x}_{s}\left(t-\tau_{s 1}\right)+B_{d} v_{c}\left(t-\tau_{i 1}\right) \\
& +h_{12}^{\prime} x_{2}\left(t-\tau_{c 2}-\tau_{i 1}\right) \\
y_{1}(t)= & C_{11} \tilde{x}_{s}\left(t-\tau_{o 1}\right)-D_{1} v_{c}\left(t-\tau_{i 1}\right)
\end{aligned}
$$


where

$$
\begin{gathered}
A_{s}=\left[\begin{array}{cccc}
1 & 0 & 0 & -0.5001 \\
0 & 0.4999 & -0.25 & -0.4999 \\
0 & 0 & 0.4999 & 0 \\
0 & 0 & 0 & 0.4999
\end{array}\right], \\
\widehat{A}_{d}=\left[\begin{array}{cccc}
0.4472 & 0 & 0 & -0.2236 \\
0 & 0.2236 & -0.1118 & -0.2236 \\
0 & 0 & 0.2236 & 0 \\
0 & 0 & 0 & 0.2236
\end{array}\right], \\
B_{d}=\left[\begin{array}{ccc}
0.4999 & 0.5001 \\
-0.25 & -0.25 \\
0.4999 & 0.4999 \\
0.4999 & -0.4999
\end{array}\right]
\end{gathered}
$$

$$
C_{11}=\left[\begin{array}{llll}
1 & 0 & 1 & 0 \\
1 & 0 & 0 & 0
\end{array}\right], \quad D_{1}=\left[\begin{array}{cc}
0 & 2 \\
0.5 & 1.5
\end{array}\right] .
$$

By OKID [21, 22] in Figure 1, the identified subsystem $S 2$ is given as

$$
\begin{aligned}
S 2: \quad x_{d 2}\left(k_{2} T_{2}+T_{2}\right)= & G_{d 2} x_{d 2}\left(k_{2} T_{2}\right) \\
& +H_{d 2} u_{d 2}\left(k_{2} T_{2}-\tau_{i 2}\right), \\
y_{d 2}\left(k_{2} T_{2}\right)= & C_{d 2} x_{d 2}\left(k_{2} T_{2}-\tau_{o 2}\right),
\end{aligned}
$$

where

$$
\begin{aligned}
& G_{d 2}=\left[\begin{array}{ccccc}
1.16 \times 10^{0} & -5.39 \times 10^{-2} & -8.39 \times 10^{-2} & 1.15 \times 10^{-2} & 1.48 \times 10^{-8} \\
5.81 \times 10^{-2} & 1.07 \times 10^{0} & -9.69 \times 10^{-3} & -8.64 \times 10^{-2} & -3.85 \times 10^{-8} \\
1.83 \times 10^{-1} & 2.44 \times 10^{-3} & 9.23 \times 10^{-1} & -5.00 \times 10^{-2} & -9.23 \times 10^{-9} \\
6.99 \times 10^{-3} & 1.90 \times 10^{-1} & 5.30 \times 10^{-2} & 7.99 \times 10^{-1} & -5.21 \times 10^{-8} \\
-8.04 \times 10^{-9} & 3.30 \times 10^{-9} & 1.38 \times 10^{-8} & -1.29 \times 10^{-8} & -7.21 \times 10^{-3}
\end{array}\right] \text {, } \\
& H_{d 2}=\left[\begin{array}{cc}
-4.08 \times 10^{-6} & 2.11 \times 10^{-4} \\
-1.46 \times 10^{-4} & 2.92 \times 10^{-4} \\
2.32 \times 10^{-5} & -2.69 \times 10^{-4} \\
1.98 \times 10^{-4} & -3.77 \times 10^{-4} \\
-6.01 \times 10^{-12} & 1.85 \times 10^{-8}
\end{array}\right] \\
& C_{d 2}=\left[\begin{array}{ccccc}
6.43 \times 10^{-1} & -8.50 \times 10^{-1} & 5.29 \times 10^{-1} & -6.39 \times 10^{-1} & -1.52 \times 10^{-8} \\
8.72 \times 10^{-1} & 7.84 \times 10^{-1} & 7.35 \times 10^{-1} & 4.80 \times 10^{-1} & -2.02 \times 10^{-8}
\end{array}\right] \text {, }
\end{aligned}
$$

in which the input time-delay $\tau_{i 2}=0.5 \times T_{2}$ and output timedelay $\tau_{o 2}=0.5 \times T_{2}$.

Following the proposed method in this paper, let the reference inputs $r(t)=\left[\begin{array}{lll}0.5 \sin (t) & 0.5 \cos (t)\end{array}\right]^{T}$ and apply them to subsystem $S 1$ and subsystem $S 2$, respectively. We obtain the observer gain matrix $L_{d}$ for $S 1$ and $S 2$ as follows:

$$
\begin{aligned}
& -1.77 \times 10^{2}-8.82 \times 10^{-2} \quad 1.96 \times 10^{0} \\
& 3.62 \times 10^{2} \quad 8.81 \times 10^{-2} \quad-3.93 \times 10^{0} \\
& \left.\begin{array}{cccc}
-1.94 \times 10^{-3} & -1.94 \times 10^{-3} & 0 & 0 \\
1.09 \times 10^{-4} & 1.15 \times 10^{-4} & 0 & 0
\end{array}\right]^{T},
\end{aligned}
$$

$S 1: \quad L_{d 1}=\left[\begin{array}{ccc}-7.19 \times 10^{-3} & -1.26 \times 10^{2} & 1.00 \times 10^{0} \\ 1.02 \times 10^{0} & 2.60 \times 10^{2} & -1.00 \times 10^{0}\end{array}\right.$

$$
\begin{aligned}
& 1.98 \times 10^{0}-1.57 \times 10^{-2}-1.26 \times 10^{2} \\
& -3.97 \times 10^{0} \quad 2.83 \times 10^{-1} \quad 2.58 \times 10^{2} \\
& 2.49 \times 10^{-1} \quad 1.97 \times 10^{0} \quad-1.23 \times 10^{0} \\
& -2.49 \times 10^{-1}-3.94 \times 10^{0} \quad 1.18 \times 10^{0} \\
& 3.25 \times 10^{-1} \quad 4.90 \times 10^{-8} \quad-1.01 \times 10^{-1} \\
& -3.67 \times 10^{-1} \quad 7.07 \times 10^{-10}-8.27 \times 10^{-2}
\end{aligned}
$$




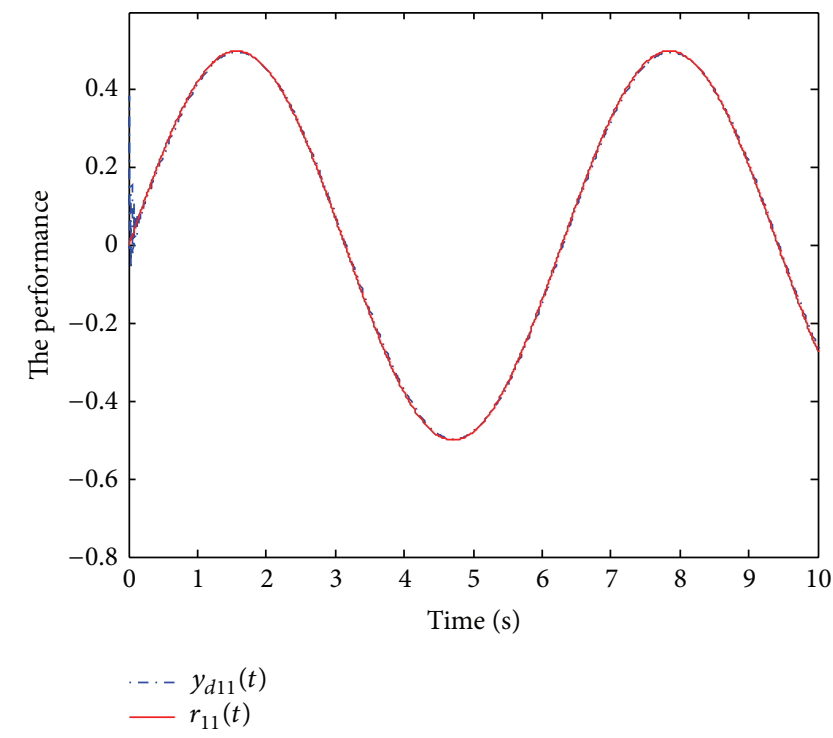

(a)

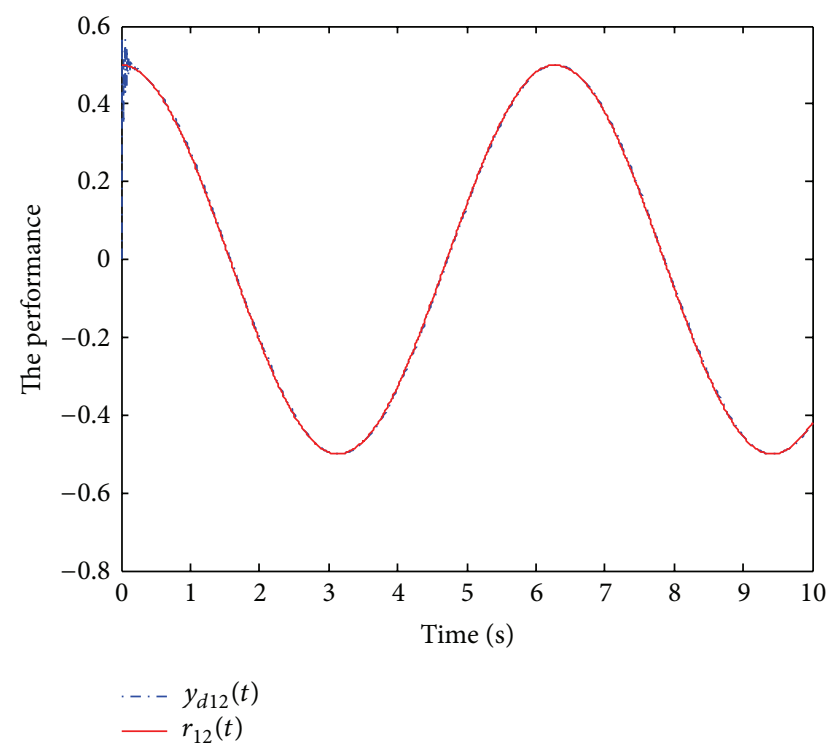

(b)

FIGURE 4: (a) Output responses of the subsystem $S 1$ : output $y_{d 11}(t)$ and reference $r_{11}(t)$. (b) Output responses of the subsystem $S 1$ : output $y_{d 12}(t)$ and reference $r_{12}(t)$.

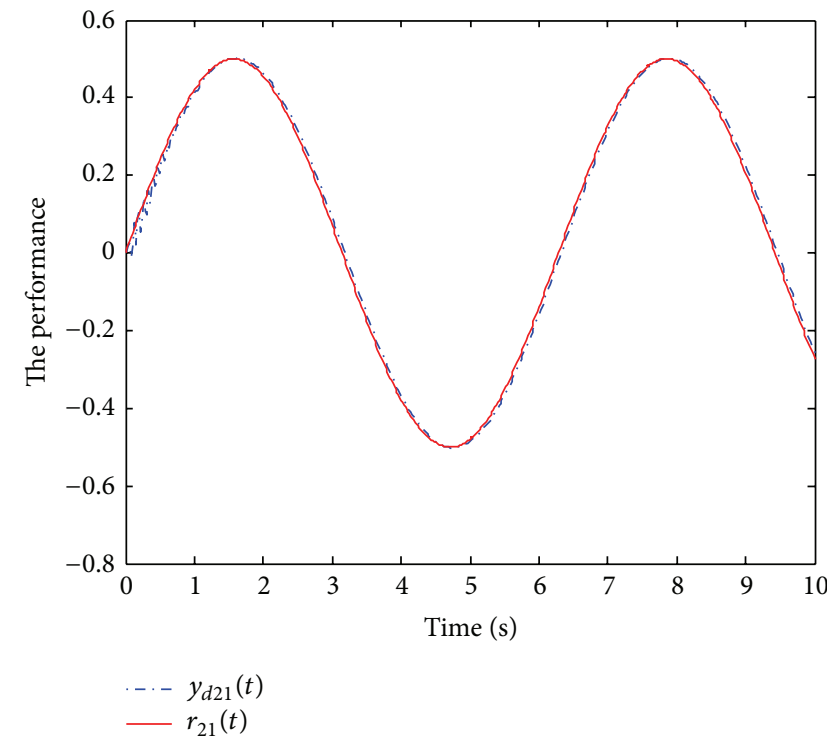

(a)

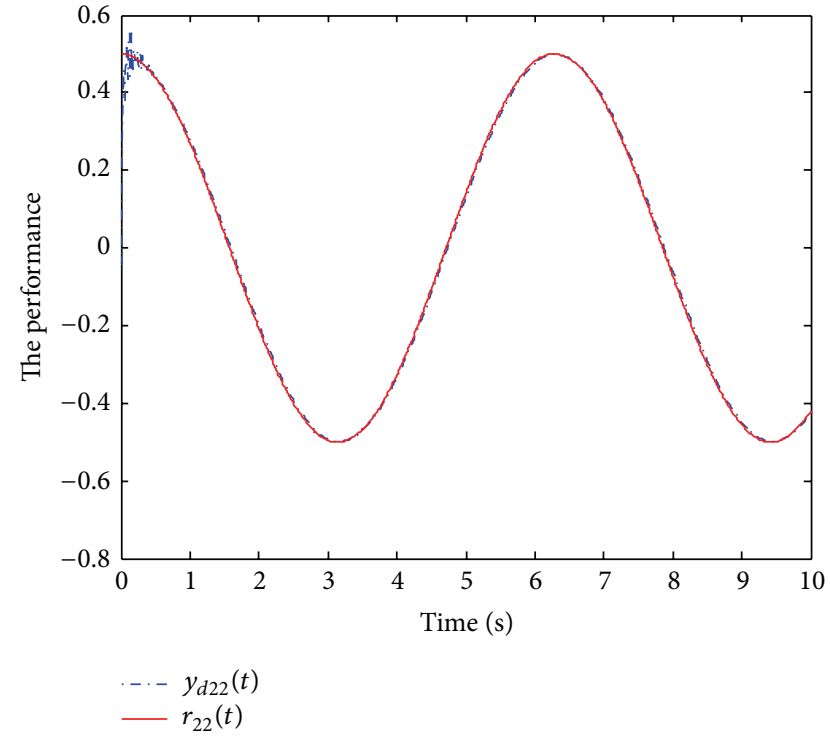

(b)

FIGURE 5: (a) Output responses of the subsystem $S 2$ : output $y_{d 21}(t)$ and reference $r_{21}(t)$. (b) Output responses of the subsystem $S 2$ : output $y_{d 22}(t)$ and reference $r_{22}(t)$.

$$
\begin{array}{lll}
-8.23 \times 10^{-2} & -1.09 \times 10^{-1} & 8.07 \times 10^{-2} \\
1.00 \times 10^{-1} & -8.55 \times 10^{-2} & -9.41 \times 10^{-2} \\
-2.15 \times 10^{-7} & 2.22 \times 10^{-2} & 3.66 \times 10^{-2} \\
-1.98 \times 10^{-8} & 2.55 \times 10^{-2} & -3.11 \times 10^{-2} \\
2.43 \times 10^{-2} & -3.02 \times 10^{-2} & -1.81 \times 10^{-6} \\
2.40 \times 10^{-2} & 2.43 \times 10^{-2} & -1.58 \times 10^{-7}
\end{array}
$$$$
\left.\begin{array}{cccc}
7.80 \times 10^{-6} & -9.87 \times 10^{-6} & 0 & 0 \\
-7.04 \times 10^{-6} & 2.65 \times 10^{-5} & 0 & 0
\end{array}\right]^{T} \text {. }
$$

Finally, the scheme of Figure 2 is implemented. For simplification, the numerical analysis is not presented and Figures 4 and 5 show the results of the simulation.

In order to confirm the independence of the control for the two subsystems, the time-varying optimal digital 


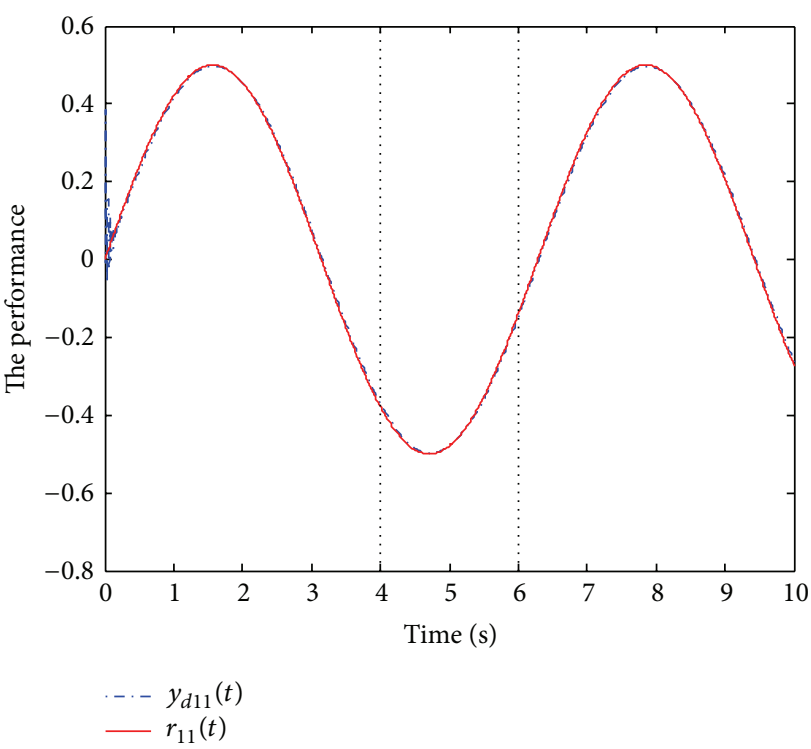

(a)

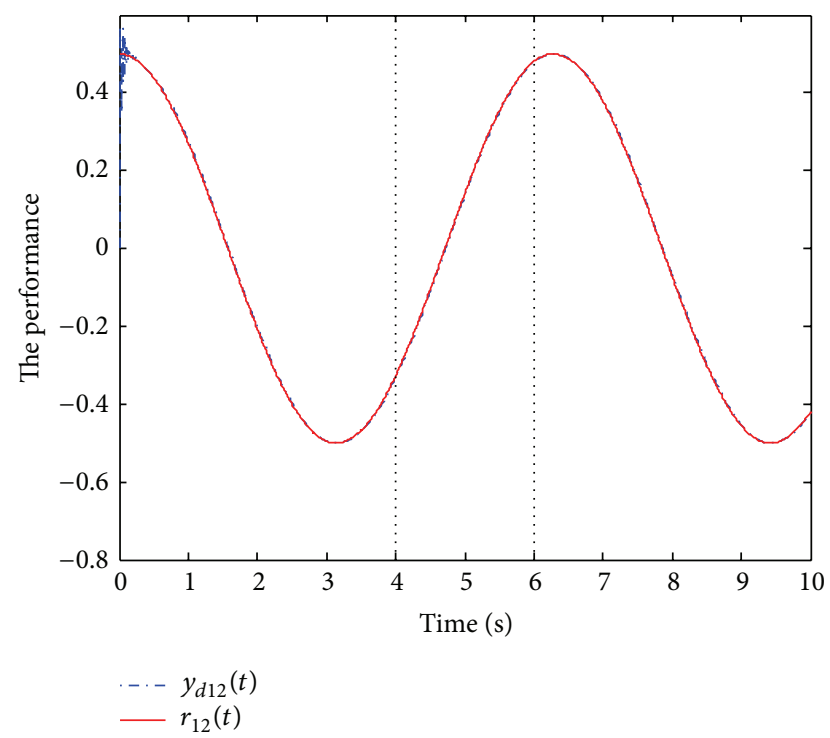

(b)

FIGURE 6: The unanticipated failure occurs without fault-tolerant control during $t=4 \sim 6 \mathrm{sec}$. (a) Output responses of the subsystem $S 1$ : output $y_{d 11}(t)$ and reference $r_{11}(t)$. (b) Output responses of the subsystem $S 1$ : output $y_{d 12}(t)$ and reference $r_{12}(t)$.

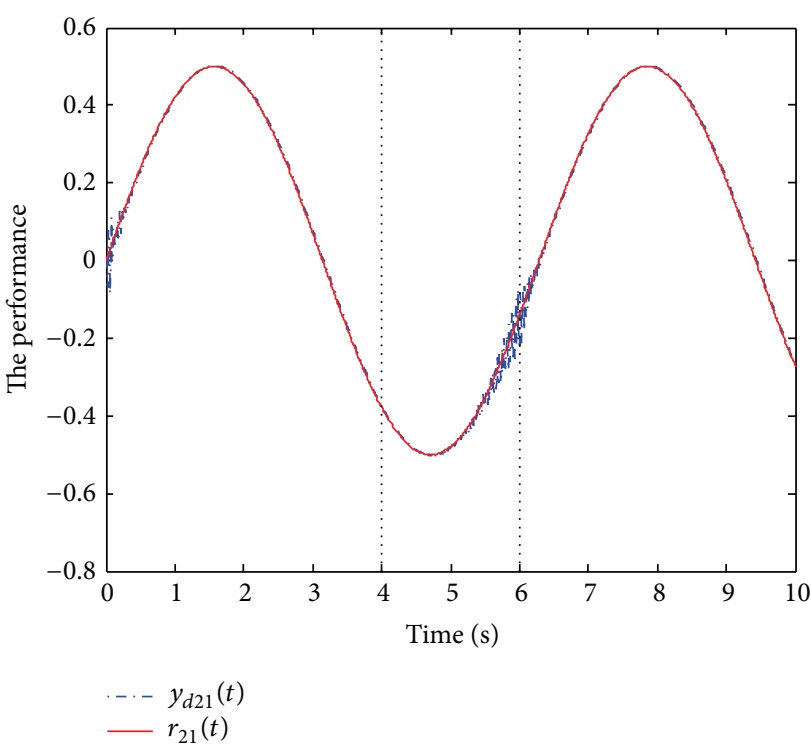

(a)

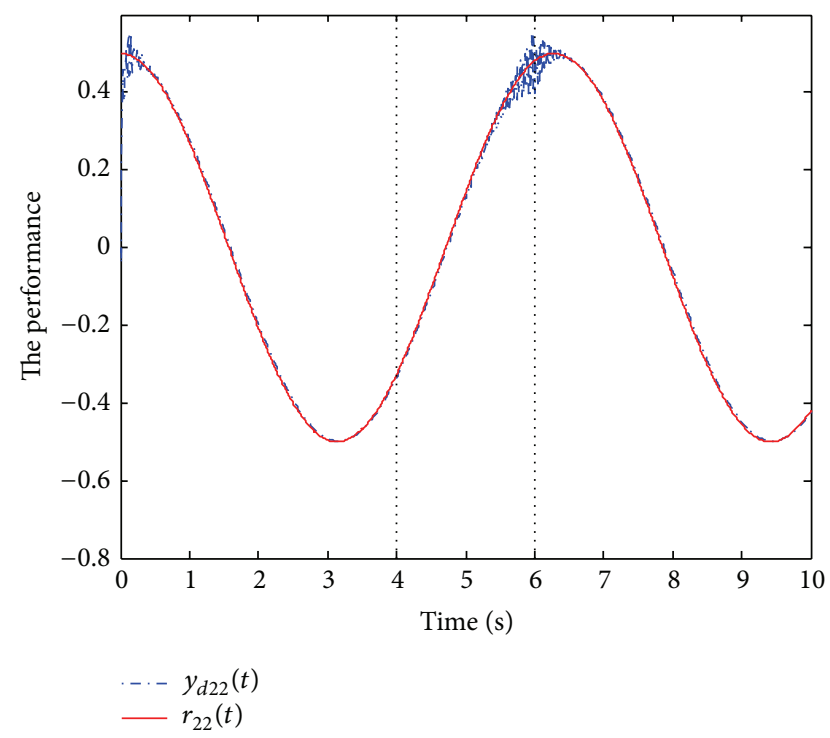

(b)

FIGURE 7: The unanticipated failure occurs without fault-tolerant control during $t=4 \sim 6 \mathrm{sec}$. (a) Output responses of the subsystem $S 2$ : output $y_{d 21}(t)$ and reference $r_{21}(t)$. (b) Output responses of the subsystem $S 2$ : output $y_{d 22}(t)$ and reference $r_{22}(t)$.

controller of the subsystem $S 2$ is reduced by multiplying a scalar 0.97 during $4 \mathrm{sec}$ to $6 \mathrm{sec}$ in this simulation. Although the time-varying optimal digital controller of the subsystem $S 2$ is reduced, the tracking performance of the subsystem $S 1$ will not be affected by this condition and the results are shown in Figures 6 and 7.

To show the effectiveness of the proposed method, we compare it with the observer/Kalman filter identification (OKID) method in the simulation for the subsystem $S 2$.
Following [20,21], let the subsystem $S 2$ be excited by the control force $u(t)$ with white noise $u(t)=\left[\begin{array}{ll}u_{1}(t) & u_{2}(t)\end{array}\right]^{T}$ having zero mean and covariance $\operatorname{diag}\left[\operatorname{cov}\left(u_{1}(t)\right) \operatorname{cov}\left(u_{2}(t)\right)\right]=$ $\operatorname{diag}\left[\begin{array}{ll}0.2 & 0.2\end{array}\right]$. Then, the comparisons between the actual outputs and the OKID method for subsystem $S 2$ are shown in Figure 8, and the comparisons between the actual outputs and the proposed method for subsystem $S 2$ are shown in Figure 9. 

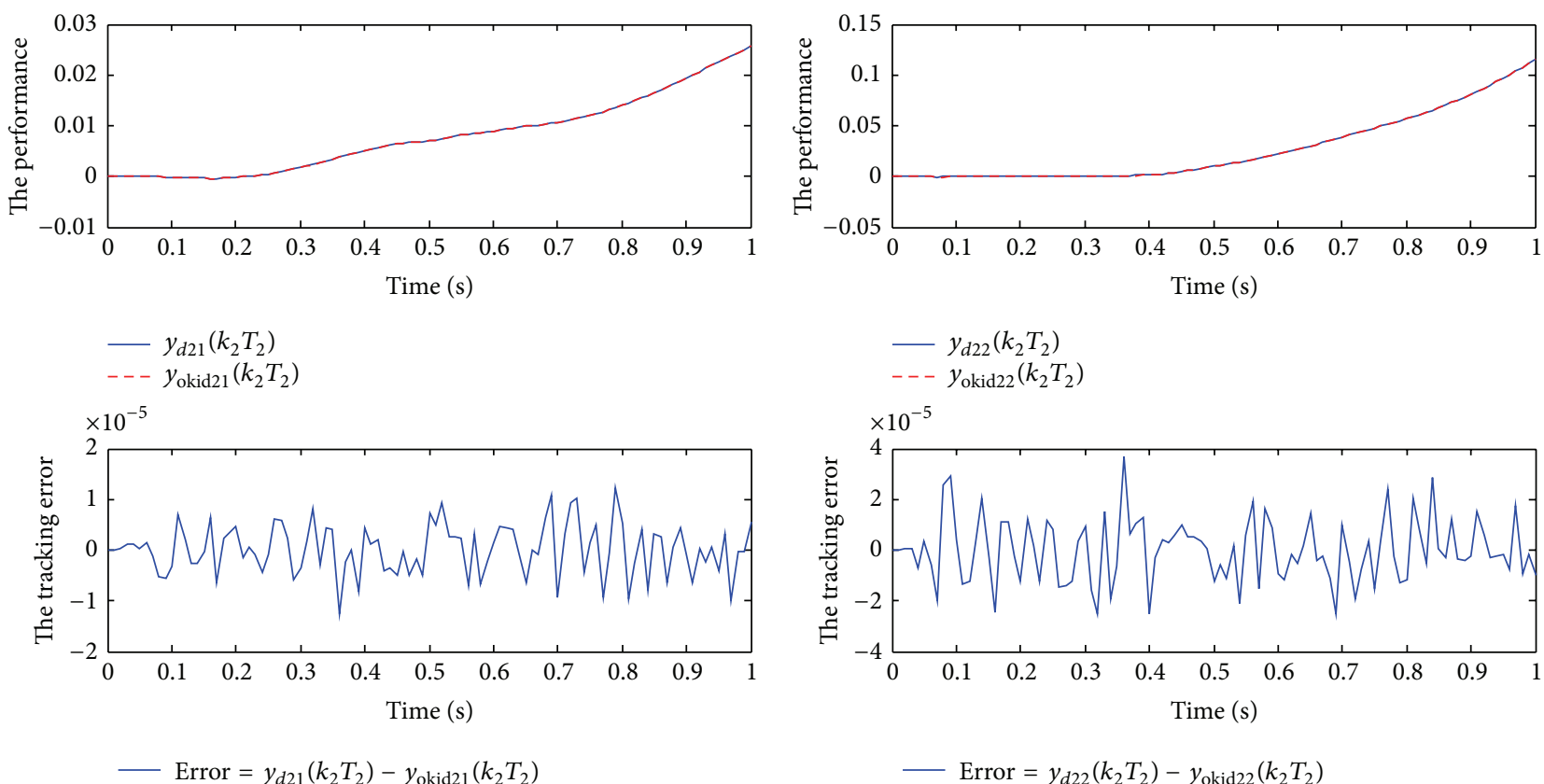

(a)

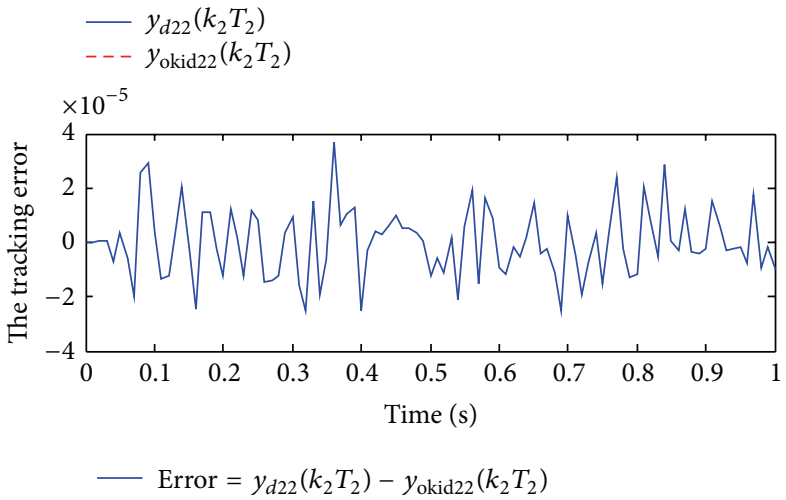

(b)

FIGURE 8: (a) The comparison between the system output $y_{d 21}\left(k_{2} T_{2}\right)$ and its observer-based output $y_{\text {okid21 }}\left(k_{2} T_{2}\right)$ by OKID for the subsystem $S 2$. (b) The comparison between the system output $y_{d 22}\left(k_{2} T_{2}\right)$ and its observer-based output $y_{\text {okid22 }}\left(k_{2} T_{2}\right)$ by OKID for the subsystem $S 2$.
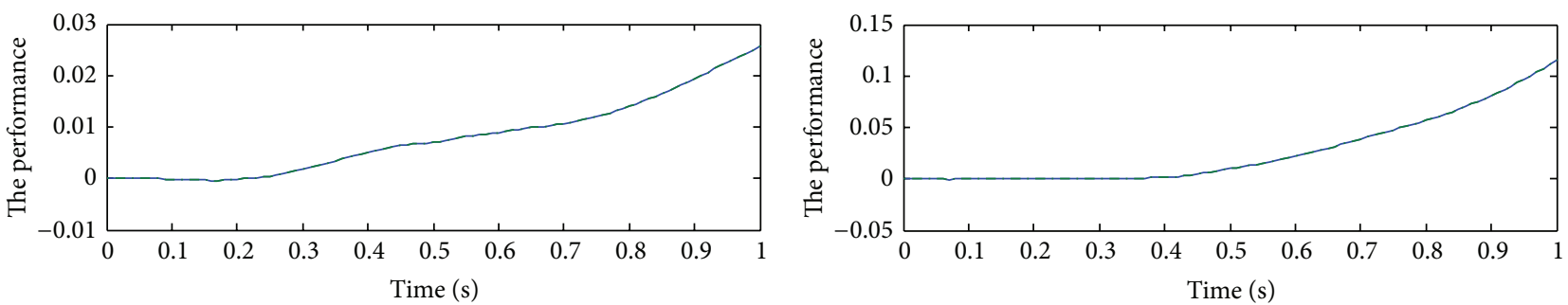

$$
\begin{aligned}
& -y_{d 21}\left(k_{2} T_{2}\right) \\
& \ldots y_{i d 21}\left(k_{2} T_{2}\right)
\end{aligned}
$$

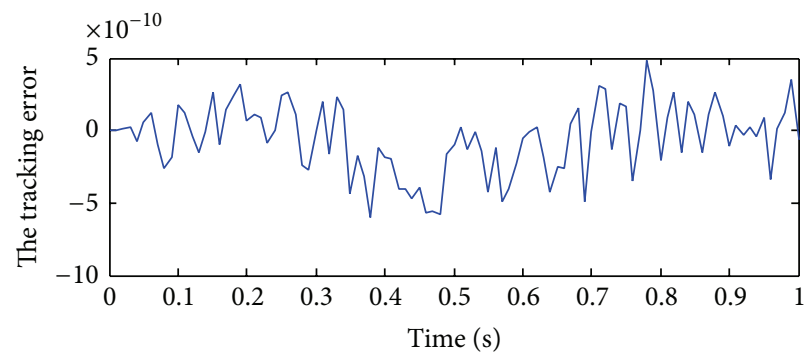

- Error $=y_{d 21}\left(k_{2} T_{2}\right)-y_{i d 21}\left(k_{2} T_{2}\right)$

(a)

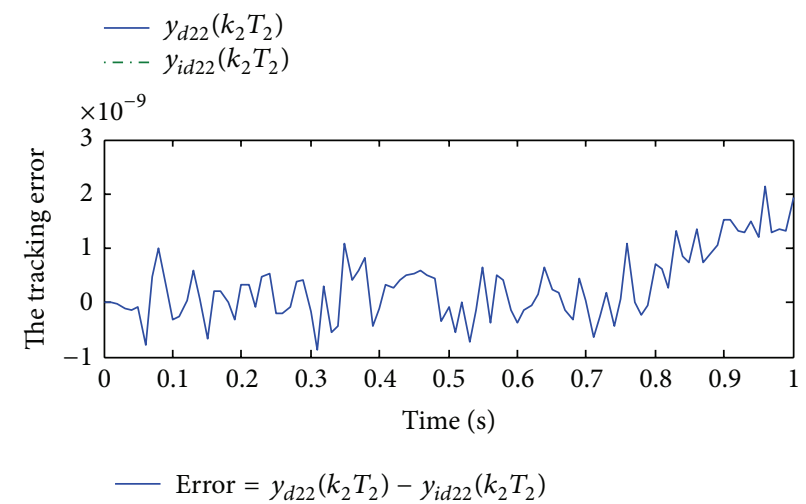

(b)

FIGURE 9: (a) The comparison between the system output $y_{d 21}\left(k_{2} T_{2}\right)$ and its observer-based output $y_{i d 21}\left(k_{2} T_{2}\right)$ by the proposed method for the subsystem $S 2$. (b) The comparison between the system output $y_{d 22}\left(k_{2} T_{2}\right)$ and its observer-based output $y_{i d 22}\left(k_{2} T_{2}\right)$ by the proposed method for the subsystem $S 2$. 
From the comparison between Figures 8 and 9, the effectiveness of the proposed method is better than OKID method in the tracking error performance.

\section{Conclusion}

This paper presents a systematical methodology of the decentralized control for the interconnected time-delay singular/nonlinear subsystems with closed-loop decoupling property. We use the observer-based suboptimal digital tracker with high gain property to keep the good tracking performance. Moreover, the decoupling property performs very well such that even if some unanticipated fault occurs in some of subsystems, it still will not affect the tracking performance of each subsystem. The proposed methods depend on the decentralized modeling of the interconnected sampled-data time-delay subsystems in Section 2 and the controller design is suitable to time-delay singular/nonlinear subsystems in Section 3. Thus, the proposed method can deal with the signal quantization and sensor delay but cannot deal with intermittent measurements and missing/fading measurements. In future works, we will pay more attention to fault-tolerant control, intermittent measurements, and missing/fading measurements by using the proposed methods.

\section{Appendix}

\section{The Decentralized Control Stabilization}

The necessary and sufficient conditions for the decentralized stabilization are presented in [29]. Here, we provide the proof for the decentralized stabilization and more details can be seen [29]. The following proofs are cited from [29].

Consider the given system $\bar{\Sigma}$ :

$$
\begin{gathered}
\bar{\Sigma}: \quad x(k T+T)=A x(k T)+\sum_{i=1}^{v} B_{i} u_{i}(k T), \\
y_{i}(k T)=C_{i} x(k T), \quad i=1, \ldots, v .
\end{gathered}
$$

The decentralized stabilization problem for $\bar{\Sigma}$ is to find controllers $\Sigma_{i}, i=1, \ldots, v$, such that the poles of the closed loop system are in the desired locations in the open unit disc. In order to provide an easier bookkeeping, we define the following matrices:

$$
\begin{array}{cc}
B=\left[B_{1} \cdots B_{v}\right], & C=\left[C_{1}^{T} \cdots C_{v}^{T}\right]^{T}, \\
K=\operatorname{diag}\left[K_{1} \cdots K_{v}\right], & L=\operatorname{diag}\left[L_{1} \cdots L_{v}\right], \\
M=\operatorname{diag}\left[M_{1} \cdots M_{v}\right], & N=\operatorname{diag}\left[N_{1} \cdots N_{v}\right] .
\end{array}
$$

Definition A.1. Consider system $\bar{\Sigma} ; \lambda \in C$ is called a decentralized fixed mode if for all block diagonal matrices $H$ one has $\operatorname{det}(\lambda I-A-B H C)=0$.

Lemma A.2. Necessary and sufficient condition for the existence of a decentralized feedback control law for the system $\bar{\Sigma}$ such that the closed loop system is asymptotically stable is that all the fixed modes of the system are asymptotically stable (in the unit disc).

Proof. We first establish necessity. Assume local controllers $\Sigma_{i}$ together stabilize $\bar{\Sigma}$ then for any $|\lambda| \geq 1$ there exists a $\delta$ such that $(\lambda+\delta) I-K$ is invertible and the closed loop system replacing $K$ with $K-\delta I$ is still asymptotically stable. This choice is possible because if $\lambda I-K$ is invertible obviously we can choose $\delta=0$. If $\lambda I-K$ is not invertible, by small enough choice of $\delta$ we can make sure that $(\lambda+\delta) I-K$ is invertible and the closed loop system replacing $K$ with $K-\delta I$ is still asymptotically stable. But the closed loop system when $K-\delta I$ is in the loop is asymptotically stable. In particular, it cannot have a pole in $\lambda$. So

$$
\operatorname{det}\left(\lambda I-A-B\left[M(\lambda I-(K-\delta I))^{-1} L+N\right] C\right) \neq 0 .
$$

Hence the block diagonal matrix $S=M(\lambda I-(K-\delta I))^{-1} L+N$ has the property that

$$
\operatorname{det}(\lambda I-A-B S C) \neq 0 .
$$

Thus $\lambda$ is not a fixed mode. Since this argument is true for any $\lambda$ on or outside the unit disc, this implies that all the fixed modes must be inside the unit disc. This proves the necessity of the Lemma A.2.

Next, we establish sufficiency. To prove that we can actually stabilize the system, we use a recursive argument. Assume the system has an unstable eigenvalue in $\mu$. Since $\mu$ is not a fixed mode there exists $N_{i}$ such that

$$
A+\sum_{i=1}^{v} B_{i} N_{i} C_{i}
$$

no longer has an eigenvalue in $\mu$. Let $k$ be the smallest integer such that an unstable eigenvalue of $A$ is no longer an eigenvalue of

$$
A+\sum_{i=1}^{k} B_{i} N_{i} C_{i}
$$

while $N_{i}$ can be chosen small enough not to introduce additional unstable eigenvalues. Then for the system

$$
\left(A+\sum_{i=1}^{k-1} B_{i} N_{i} C_{i}, B_{k}, C_{k}\right),
$$

an unstable eigenvalue is both observable and controllable. But this implies that there exists a dynamic controller which moves this eigenvalue in the open unit disc without introducing new unstable eigenvalues. Through a recursion, we can move all eigenvalues one-by-one in the open unit disc and in this way find a decentralized controller which stabilizes the system. This proves the sufficiency of Lemma A.2.

\section{Conflict of Interests}

The authors declare that there is no conflict of interests regarding the publication of this paper. 


\section{Acknowledgment}

The authors would like to thank the National Science Council of the Republic of China, Taiwan, for financially supporting this research under Contract no. NSC 101-2511-S-197-002.

\section{References}

[1] L. Dai, Singular Control Systems, Springer, Berlin, Germany, 1989.

[2] X. G. Yan, J. Lam, H. S. Li, and I. M. Chen, "Decentralized control of nonlinear large-scale systems using dynamic output feedback," Journal of Optimization Theory and Applications, vol. 104, no. 2, pp. 459-475, 2000.

[3] W.-J. Mao and J. Chu, "Robust decentralised stabilisation of interval discrete-time singular large-scale systems," IET Control Theory \& Applications, vol. 4, no. 2, pp. 244-252, 2010.

[4] F. Palacios-Quiñonero, J. Rubió-Massegú, J. M. Rossell, and H. R. Karimi, "Optimal passive-damping design using a decentralized velocity-feedback Ho approach," Modeling, Identification and Control, vol. 33, no. 3, pp. 87-97, 2012.

[5] Y. Guo, D. J. Hill, and Y. Wang, "Nonlinear decentralized control of large-scale power systems," Automatica, vol. 36, no. 9, pp. 1275-1289, 2000.

[6] D. Wang and P. Bao, "Robust impulse control of uncertain singular systems by decentralized output feedback," IEEE Transactions on Automatic Control, vol. 45, no. 3, pp. 500-505, 2000.

[7] Z. H. Guan, Y. Q. Liu, and X. C. Wen, "Decentralized stabilization of singular and time-delay large-scale control systems with impulsive solutions," IEEE Transactions on Automatic Control, vol. 40, no. 8, pp. 1437-1441, 1995.

[8] Z.-G. Wu, H.-Y. Su, and J. Chu, "Improved results on delaydependent $H_{\infty}$ control for singular time-delay systems," Acta Automatica Sinica, vol. 35, no. 8, pp. 1101-1106, 2009.

[9] C. Hua, L. Zhang, and X. Guan, "Decentralized output feedback controller design for nonlinear interconnected systems with unknown control direction and time-varying delays," International Journal of Adaptive Control and Signal Processing, vol. 28, no. 11, pp. 1160-1173, 2013.

[10] H. Wu, "Decentralized robust control for a class of largescale interconnected systems with uncertainties," International Journal of Systems Science, vol. 20, no. 12, pp. 2597-2608, 1989.

[11] S. Tong, Y. Li, and T. Wang, "Adaptive fuzzy decentralized output feedback control for stochastic nonlinear large-scale systems using DSC technique," International Journal of Robust and Nonlinear Control, vol. 23, no. 4, pp. 381-399, 2013.

[12] H. Wu, "Decentralised adaptive robust control of uncertain large-scale non-linear dynamical systems with time-varying delays," IET Control Theory and Applications, vol. 6, no. 5, pp. 629-640, 2012.

[13] J. Huang and J. I. F. Zhang, "Impluse-free output regulation of singular nonlinear systems," International Journal of Control, vol. 71, no. 5, pp. 789-806, 1998.

[14] F.-H. Hsiao, "Robustness design of fuzzy controllers for nonlinear multiple time-delay singularly perturbed systems: using dither as auxiliary," International Journal of Systems Science, vol. 44, no. 3, pp. 416-431, 2013.

[15] B. Shen, Z. Wang, J. Liang, and Y. Liu, "Recent advances on filtering and control for nonlinear stochastic complex systems with incomplete information: a survey," Mathematical Problems in Engineering, vol. 2012, Article ID 530759, 16 pages, 2012.
[16] J. Hu, Z. Wang, H. Dong, and H. Gao, "Recent advances on recursive filtering and sliding mode design for networked nonlinear stochastic systems: a survey," Mathematical Problems in Engineering, vol. 2013, Article ID 646059, 12 pages, 2013.

[17] F.-H. Hsiao, "T-S fuzzy control for dithered nonlinear singularly perturbed systems with multiple time delays," Journal of the Franklin Institute, Engineering and Applied Mathematics, vol. 349, no. 5, pp. 1828-1850, 2012.

[18] W. Zhao, X. He, and Y. Ji, "Robust $H_{\infty}$ model reduction for a class of nonlinear time-varying systems with time delays," in Proceedings of the 11th International Conference on Control, Automation, Robotics \& Vision (ICARCV '10), pp. 2391-2397, IEEE, Singapore, December 2010.

[19] J. Zhang, M. Lyu, H. R. Karimi, P. Guo, and Y. Bo, "Robust $H_{\infty}$ filtering for a class of complex networks with stochastic packet dropouts and time delays," The Scientific World Journal, vol. 2014, Article ID 560234, 11 pages, 2014.

[20] N.-T. Hu, T.-F. Wu, S. S.-D. Xu, and H.-C. Huang, "Novel observer-based suboptimal digital tracker for a class of timedelay singular systems," Journal of Applied Mathematics, vol. 2013, Article ID 150590, 19 pages, 2013.

[21] N.-T. Hu, J. S.-H. Tsai, S.-M. Guo, L. S. Shieh, and Y. Chen, "Loworder multi-rate linear time-invariant decentralized trackers using the new observer-based sub-optimal method for unknown sampled-data nonlinear time-delay system with closed-loop decoupling," Optimal Control Applications and Methods, vol. 32, no. 4, pp. 433-475, 2011.

[22] J. N. Juang, Applied System Identification, Prentice-Hall, Englewood Cliffs, NJ, USA, 1994.

[23] F. R. Ganmacher, The Theory of Matrices II, Chelsea Publishing, New York, NY, USA, 1974.

[24] R. Nikoukhah, A. S. Willsky, and B. C. Levy, "Boundary-value descriptor systems: well-posedness, reachability and observability," International Journal of Control, vol. 46, no. 5, pp. 17151737, 1987.

[25] W.-M. Wang, S.-M. Guo, and L.-S. Shieh, "Discretization of cascaded continuous-time controllers for state and input delayed systems," International Journal of Systems Science, vol. 31, no. 3, pp. 287-296, 2000.

[26] S.-M. Guo, W. Wang, and L.-S. Shieh, "Discretisation of two degree-of-freedom controller and system with state, input and output delays," IEE Proceedings-Control Theory and Applications, vol. 147, no. 1, pp. 87-96, 2000.

[27] Y.-W. Liang, S.-D. Xu, D.-C. Liaw, and C.-C. Chen, "A study of TS model-based SMC scheme with application to robot control," IEEE Transactions on Industrial Electronics, vol. 55, no. 11, pp. 3964-3971, 2008.

[28] S. S.-D. Xu, "Study of a class of T-S fuzzy-based terminal sliding mode control," Nonlinear Theory and Its Applications, IEICE, vol. 4, no. 4, pp. 375-388, 2013.

[29] M. Mahmoud, Decentralized Systems with Design Constraints, Springer, 2011. 


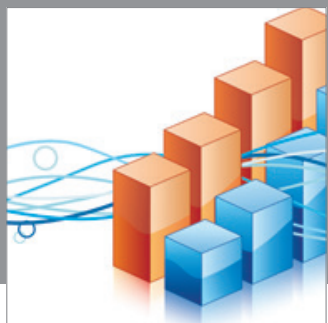

Advances in

Operations Research

mansans

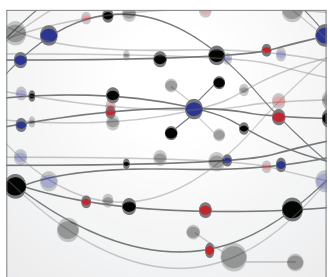

The Scientific World Journal
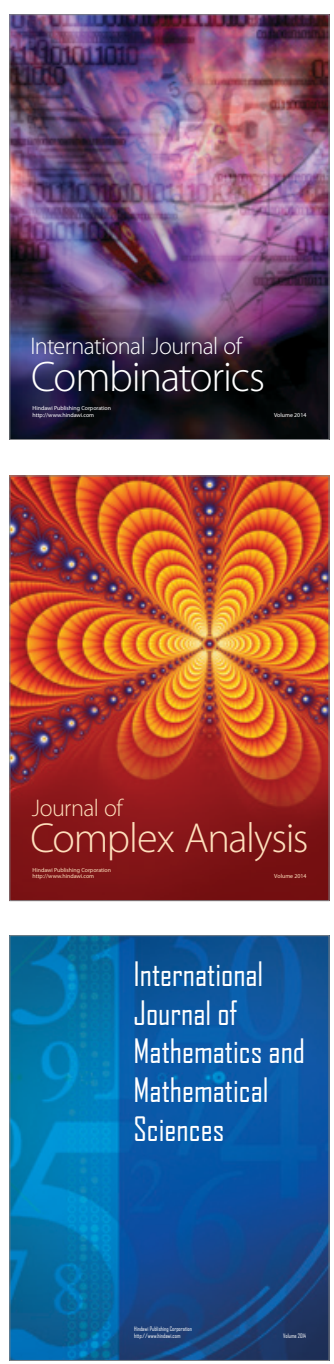
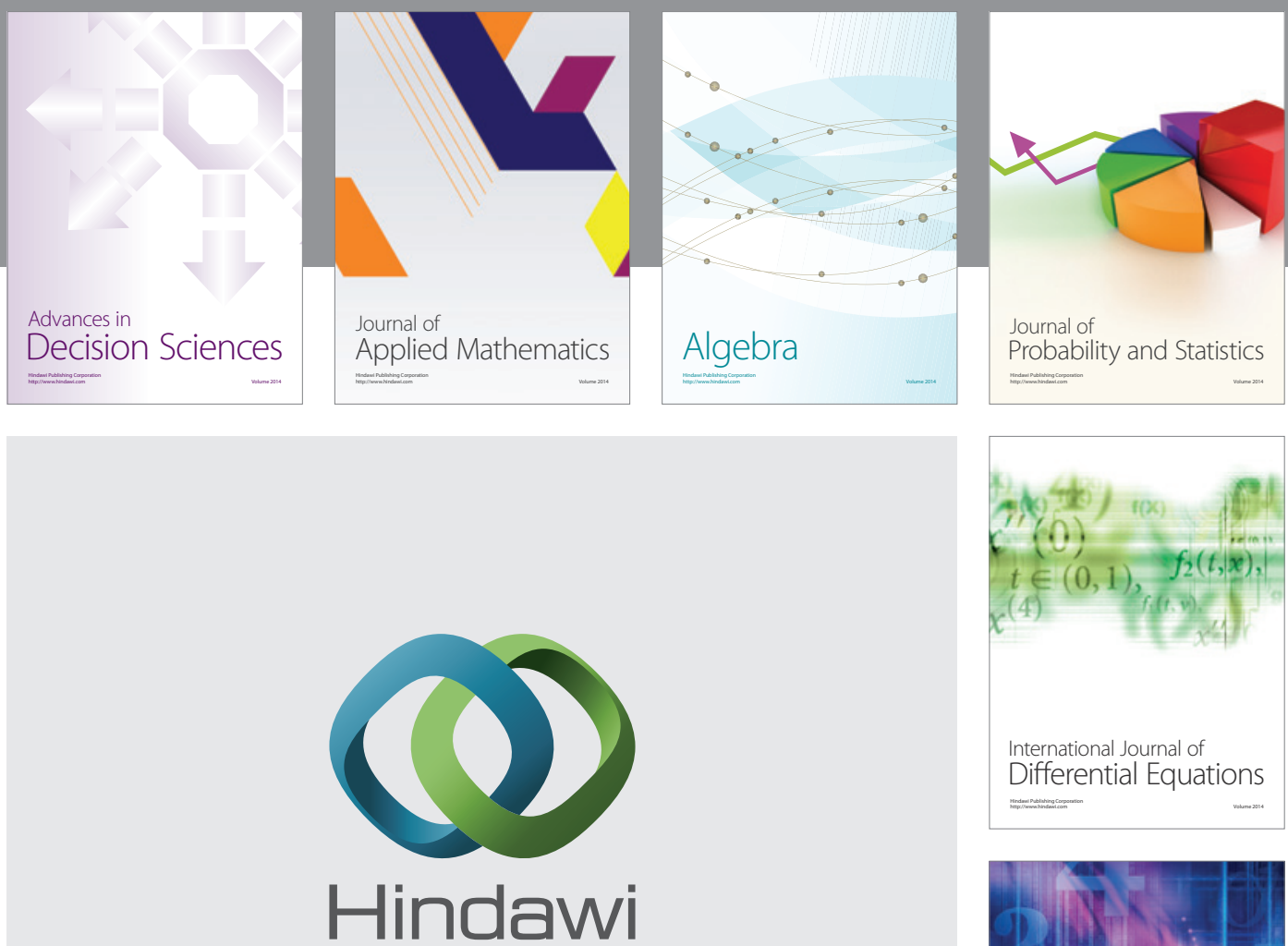

Submit your manuscripts at http://www.hindawi.com
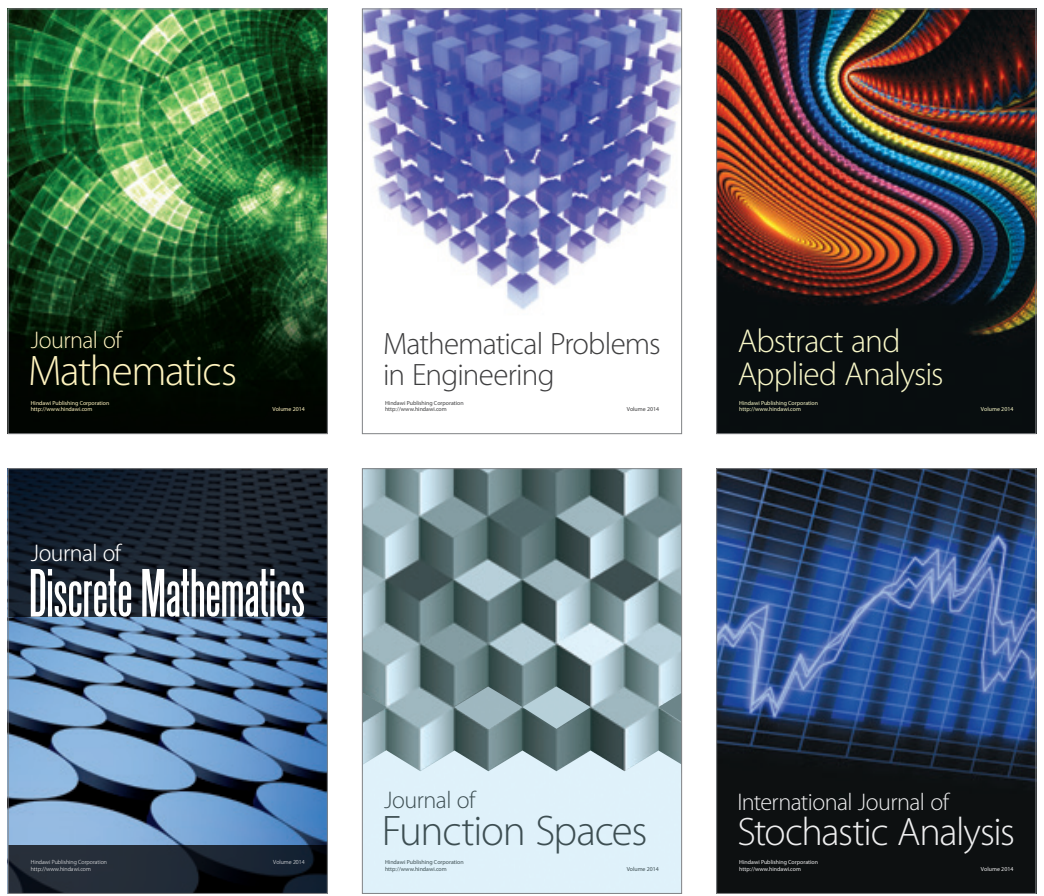

Journal of

Function Spaces

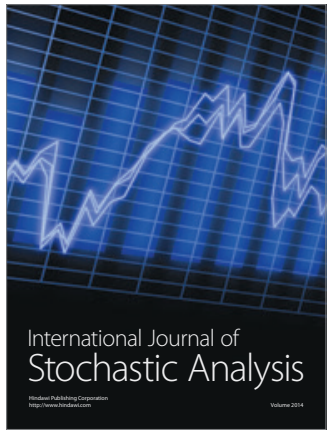

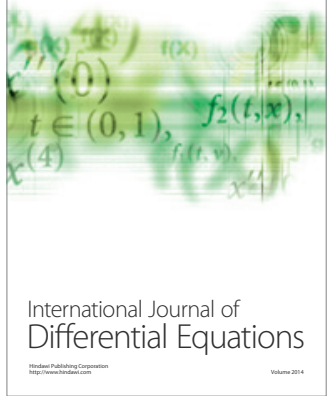
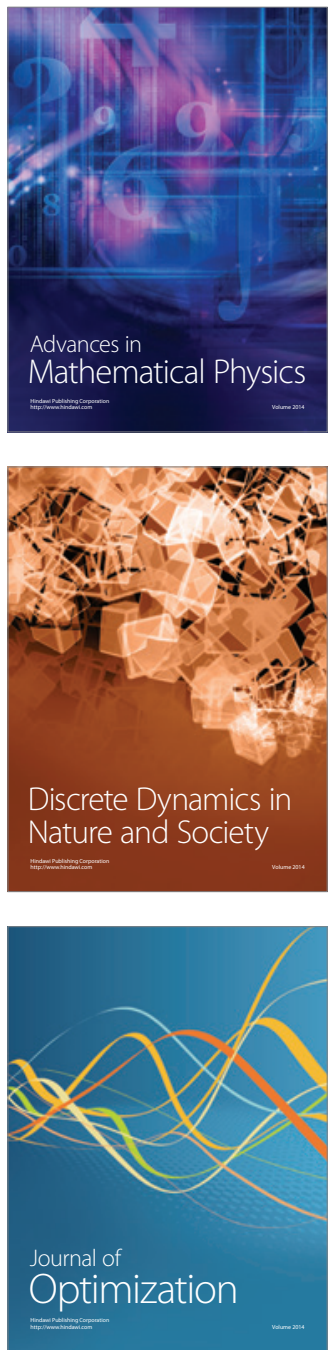